\title{
Parcs nationaux au Brésil
}

Une interprétation du contexte de la gestion et des défis en politiques publiques

National Parks in Brazil: An interpretation of the context of management and challenges in public policies

\section{Marta de Azevedo Irving}

\section{OpenEdition}

\section{Journals}

Édition électronique

URL : http://journals.openedition.org/ethnoecologie/820

DOI : 10.4000/ethnoecologie.820

ISSN : 2267-2419

\section{Éditeur}

Laboratoire Eco-anthropologie et Ethnobiologie

\section{Référence électronique}

Marta de Azevedo Irving, «Parcs nationaux au Brésil », Revue d'ethnoécologie [En ligne], 1 | 2012, mis

en ligne le 02 décembre 2012, consulté le 19 avril 2019. URL : http://journals.openedition.org/ ethnoecologie/820 ; DOI : 10.4000/ethnoecologie.820

\section{Ce document a été généré automatiquement le 19 avril 2019}

Revue d'ethnoécologie est mis à disposition selon les termes de la licence Creative Commons Attribution - Pas d'Utilisation Commerciale - Pas de Modification 4.0 International. 


\title{
Parcs nationaux au Brésil
}

\author{
Une interprétation du contexte de la gestion et des défis en politiques \\ publiques \\ National Parks in Brazil: An interpretation of the context of management and \\ challenges in public policies
}

Marta de Azevedo Irving

\section{Introduction}

1 Depuis plus d'un siècle, selon l'UNEP/CBD (2005), les pays du monde entier possèdent des réserves de protection des ressources naturelles ayant une valeur paysagère ou une importance pour la gestion de la biodiversité. Dans ce contexte, les aires protégées, et en particulier les parcs nationaux, sont reconnus comme des outils-clés pour la protection de la nature. Cependant, ces quarante dernières années ont vu un changement de paradigme de l'interprétation du rôle des aires protégées, évoluant d'une conception originelle de "parcs nationaux et réserves» vers une approche plus large en termes conceptuels et appliqués, avec également une attention particulière à la possibilité d'utilisation durable.

2 Selon Diegues (1996), les parcs nationaux ont été créés pour protéger la vie sauvage, menacée par la «civilisation urbano-industrielle, destructrice de nature », selon une interprétation qui illustre clairement un processus moderne de séparation nature-société. Par ailleurs, Van Schaik et Rijksen (2002) affirment que les parcs sont considérés comme « vitaux pour la perpétuation de la biodiversité dans un monde dominé par l'homme ». Mais selon l'UNEP/CBD (2005), les aires protégées doivent avoir de la valeur, non seulement dans leur fonction originelle de conservation des ressources naturelles, mais également pour le bien-être humain, la réduction de la pauvreté et le développement durable. Ainsi, les aires protégées, et spécialement les parcs nationaux, ont, en théorie, un rôle fondamental, à la fois pour la protection de la diversité génétique, mais également pour l'amélioration de la qualité de vie des populations locales, le développement du 
tourisme et des loisirs, et la répartition des bénéfices résultant des processus de protection et d'utilisation de la biodiversité.

Globalement, selon cette même source, le nombre d'aires protégées a crû significativement durant la dernière décennie, avec actuellement plus de cent mille aires protégées, qui couvrent approximativement $12 \%$ de la superficie de la planète. Elles sont ainsi devenues un des principaux « usages » de la terre. Cependant, bien que le nombre des aires dédiées à la protection de la nature soit en augmentation progressive, la diversité biologique continue à être menacée, ce qui indique que le système global des aires protégées paraît inadéquat pour accomplir sa fonction, et ce pour diverses raisons.

Ainsi, selon l'optique contemporaine, le "mythe moderne de la nature inviolée " (Diegues 1996) perd progressivement de sa signification. Cela amène un nouveau regard qui considère la nature liée à sa composante humaine et sociale, en fonction des questions éthiques sous-jacentes à l'équilibre à trouver entre protection de la nature et inclusion sociale.

5 Cette discussion a été conduite lors de la septième conférence sur la Convention de la diversité biologique (COP 7), en considérant le Millenium Development Goals et le Plan d'application du Forum mondial de développement durable. Cette conférence a adopté un programme global de travail pour les aires protégées, structuré en trois phases :

- génération d'un Plan stratégique (Master Plan);

- établissement de mécanismes pour gérer les principales menaces et pour garantir des ressources financières et politiques en vue de la participation des communautés locales et indigènes et pour la répartition des bénéfices provenant de la protection de la nature ;

- consolidation d'un système global d'aires protégées, géré efficacement, et son intégration à grande échelle pour envisager des espaces plus larges, dans des environnements terrestres ou marins.

6 Ainsi, la notion d'aires protégées se déplace, progressivement, de buts strictement préservationnistes vers des modèles d'usage plus flexible des ressources naturelles renouvelables, incluant la socio-biodiversité. En conséquence, la gestion des parcs nationaux est rediscutée en fonction du contexte socioéconomique dans leurs régions d'insertion, selon une approche de mosaïques et de développement régional. Cependant, ce thème suscite des débats permanents au Brésil et, selon Dourojeanni et Pádua (2001: 132).

« Entre la croissance de la population, la pauvreté, les inégalités, l'exclusion sociale et la dégradation environnementale, il existe une relation grandement complexe, dans laquelle il est toujours difficile d'identifier cause et effet. Néanmoins, le résultat est toujours le même... augmentation de la pression sur les espaces naturels, sur les ressources naturelles et, d'une manière ou d'une autre, sur les unités de conservation (UC) ${ }^{1}$ "

7 Mais quelle que soit la lecture, le concept de biodiversité (et ses développements) se situe au centre des débats mondiaux et au Brésil en particulier, et implique de multiples dimensions et perceptions: la préoccupation de la survie humaine, l'émergence de nouveaux modèles de développement, la dynamique des relations internationales, et de nouvelles approches de politiques publiques (Bensusan 2002).

8 En réponse aux défis établis par la Convention sur la diversité biologique, un Système national d'unités de conservation de la nature (SNUC) ${ }^{2}$ a été institué au Brésil en 2000 (Brasil 2000,2002 ). Il décrit les typologies et catégories de gestion des aires protégées et établit les critères et les normes pour leur création, leur implantation et leur gestion. En 2006, le 
Plan stratégique national des aires protégées (PNAP) ${ }^{3}$ a été établi avec l'objectif de consolider les stratégies de protection de la biodiversité pour les aires protégées, comme résultat direct d'un débat national impliquant le secteur public et d'autres secteurs de la société brésilienne.

9 Cependant, la discussion et la consolidation du système (dans sa forme légale) ont duré pratiquement dix ans et ont reflété une grande controverse conceptuelle et idéologique dans l'interprétation de la société brésilienne sur le rôle des aires protégées. Elles se sont manifesté d'un côté, par la perspective préservationniste influencée directement par le «modèle Yellowstone» et, d'un autre côté, par la réalité historique de l'occupation humaine de ces espaces, comme discuté entre autres par Diegues (1996) et Irving (2002). À la suite de ce processus de négociation, le SNUC a considéré deux types d'aires protégées : «Protection intégrale» (catégorie dans laquelle sont insérés les parcs nationaux) et «Usage durable».

10 Ainsi, le présent travail examine, de manière préliminaire, le contexte de gestion des parcs nationaux du Brésil, en gardant à l'esprit les défis de la Convention sur la diversité biologique et les instruments récents de politiques publiques: le Système national des unités de conservation (SNUC) et le Plan stratégique national des aires protégées (PNAP).

\section{Concept et origine des parcs nationaux au Brésil}

11 Selon le SNUC (Brasil 2000), dans son article 11, un parc national a comme objectif formel :

«... la préservation des écosystèmes naturels de grande importance écologique et de beauté scénique permettant la réalisation de recherches scientifiques, le développement d'activités d'éducation et d'interprétation environnementales, les loisirs en contact avec la nature, et le tourisme écologique ...»

12 Dans cette perspective, la gestion des parcs nationaux entre dans la catégorie de protection intégrale où sont interdites l'utilisation directe des ressources naturelles renouvelables et l'occupation humaine. Les parcs sont donc des aires protégées essentielles face aux défis de l'application du PNAP, du fait qu'ils sont indispensables aux engagements globaux de conservation de la biodiversité, qu'ils ont une reconnaissance et une diffusion internationales, et qu'ils illustrent, comme aucune autre aire protégée, les défis pour la gestion, en prenant en compte l'histoire de la séparation nature-société. Ainsi, la gestion des parcs nationaux au Brésil demande la construction d'une nouvelle conception de protection de la nature fondée sur des engagements prenant en compte la dimension sociale.

13 L'origine des parcs nationaux au Brésil est le résultat historique d'un modèle d'occupation du territoire fondé sur la conception d'une nature illimitée et de la dissociation entre nature et société. L'attention particulière portée aux implications écologiques de ce modèle est pourtant récente (Pádua 2004). Selon cet auteur, la relation nature-société était basée sur un modèle d'utilisation du territoire à des fins économiques, c'est-à-dire avec le postulat que les ressources naturelles devaient être explorées économiquement, que la biodiversité pouvait être négligée dans la planification de l'aménagement du territoire et que l'investissement en espèces exotiques pour la monoculture était nécessaire, comme l'illustre le paragraphe suivant (Pádua 2004 : 13) :

"Quand les colonisateurs portugais ont commencé à arriver sur le territoire brésilien, à partir de 1500, ils ont rencontré une combinaison impressionnante de 
mangroves, forêts, champs et autres structures complexes produites par la dynamique de la nature. [...] Les conquérants se sont tout de suite aperçu que l'exploration directe de la nature serait le principal axe de recherche des richesses dans cette partie de l'Amérique. »

Dans ce contexte historique de l'exploration illimitée de la nature, les premières préoccupations sur la conservation de la nature au Brésil ont seulement commencé à partir du XVIII ${ }^{\mathrm{e}}$ siècle (Pádua 2004). La première aire protégée du pays, le Parc national d'Itatiaia, s'est inspirée du modèle de "Yellowstone ${ }^{4}$ qui a influencé la logique contemporaine de protection de la nature. Le Parc national d'Itatiaia a été créé en $1937^{5}$, trois ans après la promulgation, en 1934, du Code forestier brésilien, qui a introduit le concept d'aire protégée sous l'influence directe du contexte international. Selon Irving (2000), l'institutionnalisation politique et administrative de la protection de la nature, consolidée pendant la première moitié $\mathrm{du} \mathrm{xx}^{\mathrm{e}}$ siècle, résulte du contexte international, mais également d'un lent et large processus de valorisation de la nature, influencé par divers secteurs de la société brésilienne.

15 En 1965, un nouveau code forestier définit les parcs nationaux comme des aires spécifiquement dirigées vers la protection des attributs exceptionnels de la nature, en conciliant la protection de la flore, de la faune et des beautés naturelles, avec des objectifs éducatifs, récréatifs et scientifiques. Selon cette chronologie, le règlement des parcs nationaux (encore en vigueur) fut institué en 1979 (Bensusan 2006). Plus récemment, la Constitution Fédérale de 1988 attribue la responsabilité de la protection du patrimoine naturel au pouvoir public et à toute la société brésilienne. Mais c'est le Système national des unités de conservation (Brasil 2000, 2002) qui établit la conception définitive pour les parcs nationaux, selon la perspective d'un système intégré et une stratégie d'aires protégées, en considérant les engagements globaux et les avancées en accord avec la Convention sur la diversité biologique.

La création de parcs nationaux au Brésil reflète ainsi, de manière évidente, le développement de stratégies nationales de protection de la nature qui, bien que significativement innovantes, ont historiquement privilégié dans certains cas des modèles centralisateurs d'implantation et de gestion. Trois étapes sont identifiées pour ce processus (Medeiros et al. 2004) :

- les années 1930 voient la définition des premiers instruments législatifs liés à la création d'aires protégées et l'établissement du premier parc national du pays, dans un mouvement de protection des écosystèmes brésiliens, selon une logique qui renforce et centralise le rôle de l'État dans la gestion de la biodiversité;

- la période de la dictature militaire entraîne la révision et la définition de nouveaux instruments de politiques publiques, ainsi que du point de vue géopolitique un regard plus national, engageant des actions de contrôle du territoire et la définition d'une base institutionnelle et légale pour les politiques environnementales ;

- la période post-1985 correspond à la redémocratisation du pays, ainsi qu'à la potentialisation et à la restructuration des stratégies de protection de la nature, avec une tendance claire vers une vision systémique. Cette phase culmine avec la création, en 2000, du Système national des aires protégées, qui définit les instruments de participation sociale à la gestion. Ensuite, l'établissement du Plan stratégique national des aires protégées, en 2006, consolide les principes et directives pour mettre en place les engagements du pays visà-vis de la Convention sur la diversité biologique et aux enjeux liés à la réduction des inégalités sociales. 
Dias (1994) résume aussi la motivation pour la création d'aires protégées au Brésil, en trois périodes distinctes :

- les années 1970, centrées sur la protection des écosystèmes représentatifs de la biodiversité brésilienne ;

- les années 1980, qui ont privilégié la conservation de la biodiversité, pour son utilisation potentielle en biotechnologie et pour la gestion des fonctions écologiques ;

- les années 1990, avec la conservation de la biodiversité dans différents systèmes économiques de production durable.

Mais l'origine du modèle brésilien est partie d'hypothèses inadéquates sur la réalité du pays, parce que le «modèle Yellowstone » privilégie la notion de wilderness et part du principe de l'existence d'espaces vides, sans présence humaine, comme présupposé pour la protection de la nature. Cette conception a entrainé divers conflits et la consolidation de pratiques de gestion centralisée, dans lesquelles le pouvoir public fut l'unique responsable du processus. Ce contexte fut modifié seulement à partir de la Politique nationale de l'environnement (1981), du Système national des unités de conservation (2000) et, plus récemment, du Plan stratégique national des aires protégées, pour citer seulement les instruments législatifs les plus importants pour la thématique en question.

Dans le cas brésilien, selon Dourojeanni et Pádua (2001), la conservation de la biodiversité, en particulier pour les aires protégées en protection intégrale comme les parcs nationaux, représente une source de divergences entre environnementalistes et socio-environnementalistes. Pour les premiers, s'il n'existait pas d'unités de conservation de protection intégrale, les pertes de biodiversité seraient significativement plus importantes que celles qui sont observées. Les socio-environnementalistes rejettent cette position, en affirmant que la coexistence entre homme et nature peut être harmonieuse et sans perte de biodiversité. De plus, selon ces auteurs, "prétendre démontrer que la présence des activités humaines n'a pas d'impact négatif sur la nature s'apparente à occulter la lumière du soleil avec la main » (Dourojeanni \& Pádua 2001 : 69). Il est évident, cependant, que l'interprétation symbolique des actions prédatrices de l'homme sur la nature, récurrente depuis le XVIII ${ }^{\mathrm{e}}$ siècle parmi théoriciens, chercheurs et gestionnaires, est bien présente sur la scène contemporaine.

Pour toutes ces raisons, les parcs nationaux représentent un défi majeur pour la mise en œuvre de nouvelles pratiques en politiques publiques de conservation de la biodiversité et pour la mise en place d'une gouvernance démocratique (Irving et al. 2006).

\section{Contexte et gestion des parcs nationaux au Brésil}

21 Il existe actuellement au Brésil 55 parcs nationaux (IBAMA 2006), dans différents écosystèmes. Ils représentent plus de $20 \%$ du territoire national légalement protégé, ce qui équivaut à peu près à 17631180 hectares. Mais les parcs ne sont pas distribués de manière homogène ; ils sont localisés dans différents types d'écosystèmes et sont soumis à différents niveaux de pression anthropique. La majeure partie des parcs nationaux est concentrée, dès l'origine, dans la forêt atlantique, région également soumise au niveau le plus élevé de pression. Mais ces dernières années, la création de nouveaux parcs nationaux est surtout concentrée en Amazonie qui, à ce jour, compte 13 parcs nationaux représentant une surface de 13417384 hectares. 
Le processus de création de parcs nationaux ne fut pas continu et n'a pas résulté des mêmes priorités stratégiques des différents gouvernements (Figure 1).

Figure 1. Évolution chronologique du processus de création des parcs nationaux (en nombre)

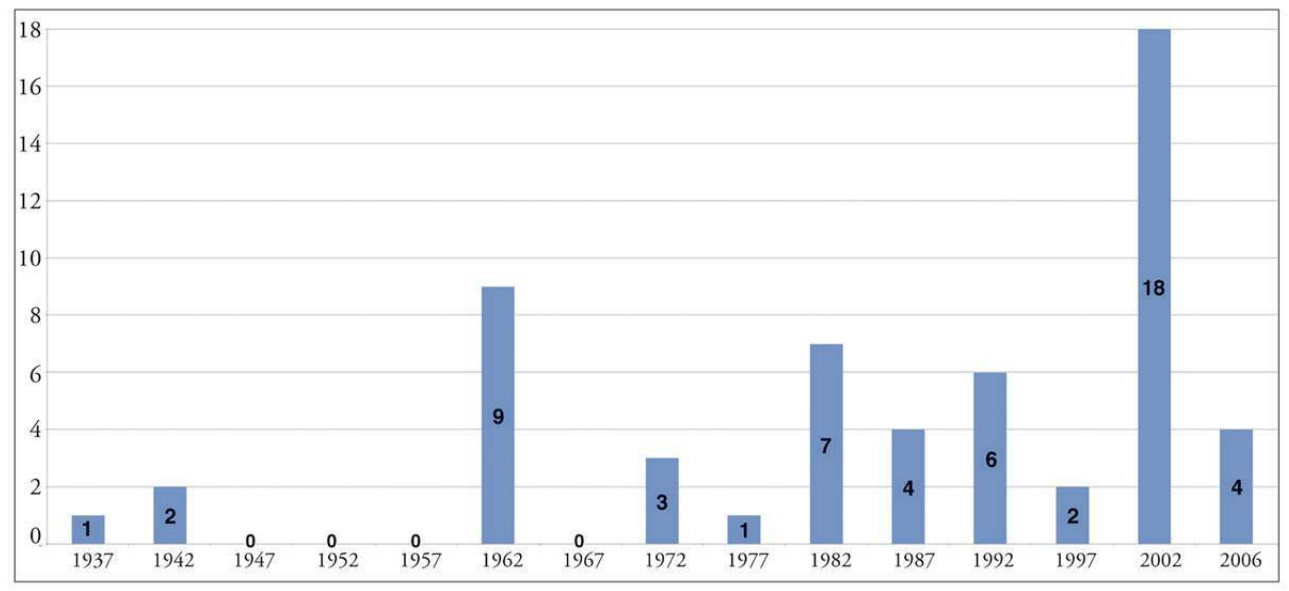

(D'après IBAMA 2006)

Cependant, de nouvelles parcelles du territoire national ont été progressivement agrégées à ce statut de protection, comme l'illustre la figure 2.

Figure 2. Évolution chronologique du processus de création des parcs nationaux (en hectares)

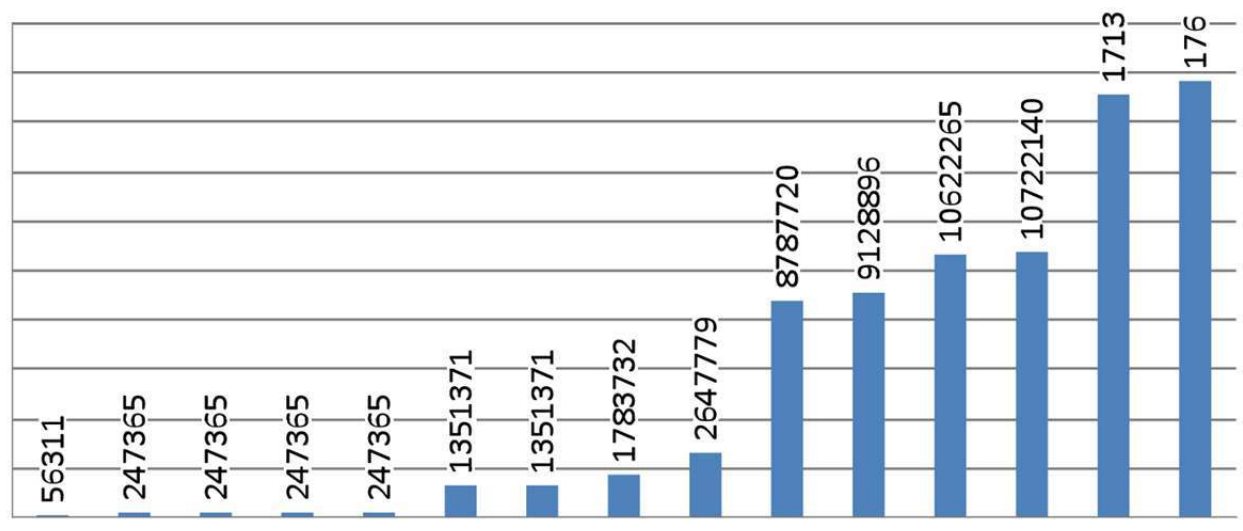

(D'après IBAMA 2006)

Il faut également mentionner que la création de parcs nationaux au fil des ans n'a pas été accompagnée par une prise en compte des facteurs sociaux, capables de promouvoir la participation de la société à la discussion de politiques publiques de protection de la nature. Ceci a probablement été la cause d'une grande partie des conflits liés à l'existence de parcs nationaux au Brésil (Irving et al. 2006).

De la même manière, la création de parcs ne fut pas accompagnée par une consolidation des stratégies de gestion. Parmi les instruments de gestion prévus par le SNUC, deux sont prioritaires comme mécanismes potentiels pour l'inclusion et le contrôle sociaux, et donc pour la participation de la société au processus de prise de décision : le plan de gestion et le conseil de gestion.

Selon le SNUC, le plan de gestion est défini comme : 
«... un document technique selon lequel s'établissent une zone tampon et des normes qui doivent présider à l'utilisation des aires protégées et à la gestion des ressources naturelles, en incluant l'implantation de structures physiques nécessaires à la gestion. » (Brasil 2000).

Selon l'interprétation du SNUC (Brasil 2000), le plan de gestion peut être élaboré par l'organisme responsable, avec ou sans participation de la communauté intéressée, suivant la catégorie de gestion. Ainsi, cet instrument de gestion peut être interprété, en théorie, comme une opportunité pour l'échange de savoirs et la construction collective et réaliste de pactes sociaux.

\section{, avec les fonctions suivantes :}

- élaboration d'un règlement interne, dans un délai de quatre-vingt-dix jours à partir de son installation;

- accompagnement, élaboration, application et révision du plan de gestion (quand c'est pertinent), pour garantir son caractère participatif ;

- intégration de l'unité de conservation à d'autres unités et espaces territoriaux protégés adjacents, ainsi qu'à sa zone tampon ;

- intégration des intérêts des divers secteurs sociaux;

- évaluation du budget et du rapport financier annuel élaboré par l'organe exécuteur ;

- position de principe face à des activités potentiellement problématiques pour l'environnement à l'intérieur de l'aire protégée, de sa zone tampon, des mosaïques, ou des corridors écologiques de la région ;

- proposition de directives et d'actions pour rendre compatibles l'intégration et l'optimisation de la relation avec la population à l'intérieur de l'unité de conservation ou de sa zone tampon.

29 Ainsi, le conseil représente un instrument de gestion et une instance potentielle pour la mise en place d'une gouvernance, d'une expression locale, de la représentation et de la participation de la société, rendant possible, en théorie, l'exercice de la citoyenneté. Il constitue un "espace potentiel » de médiation dans les relations société-État, et peut représenter une voie importante de changements sociaux et de démocratisation. En conséquence, le conseil est l'espace institutionnel pour l'engagement de la société dans les processus de prise de décision, non seulement pour les actions de protection de la nature, mais également, en principe, pour la planification et la gestion démocratique de la biodiversité.

Cependant, en 2006, la moitié seulement des parcs nationaux avaient élaboré leurs plans de gestion ou installé leurs conseils de gestion ${ }^{7}$ (Figure 3). 


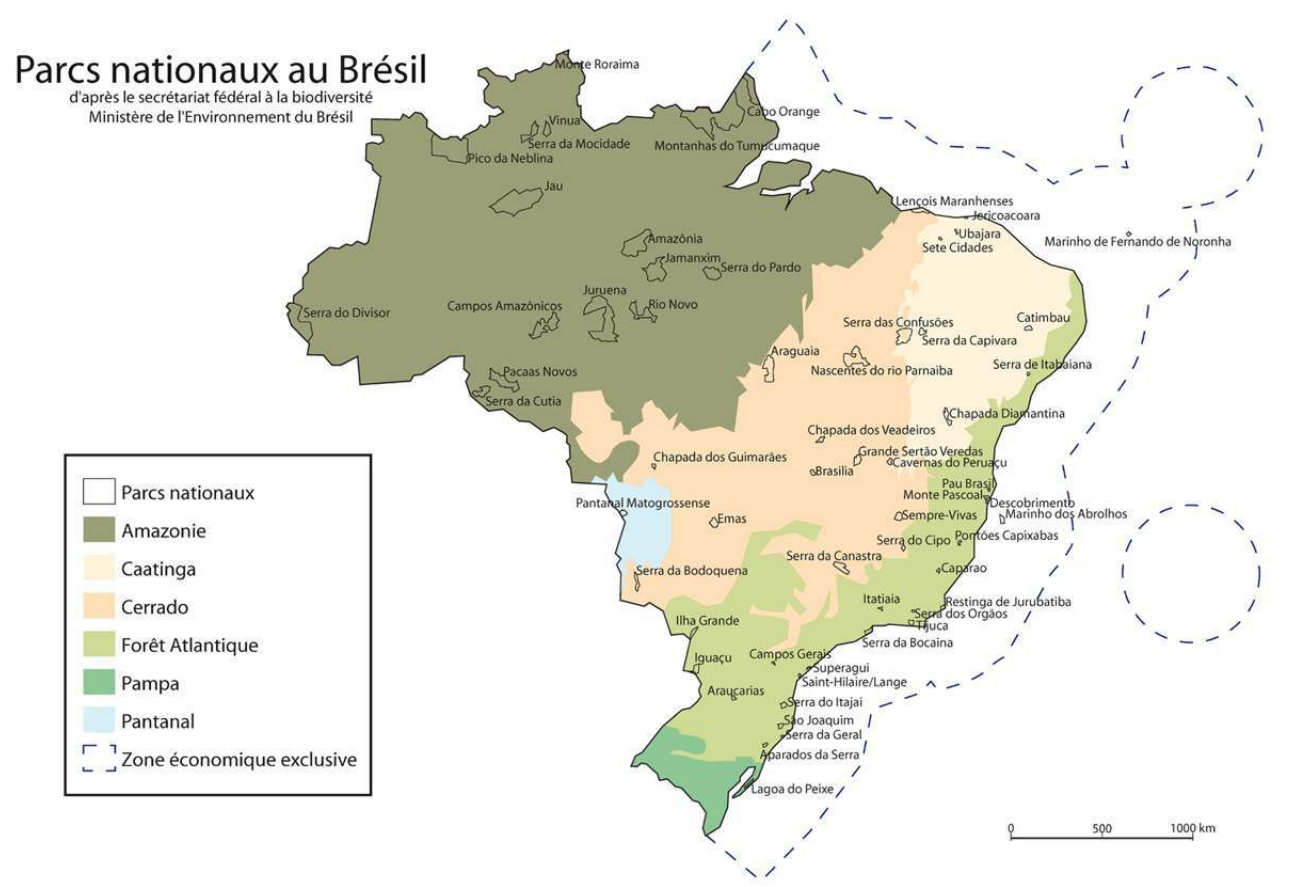

Évidemment, l'« effet SNUC » devrait pouvoir inverser cette tendance, mais le processus est lent $\mathrm{du}$ fait: de ressources humaines et financières limitées; d'une articulation contradictoire en matière de politiques publiques; et de la difficulté d'appliquer la gestion des aires protégées dans un contexte de développement régional fortement influencé par les demandes sociales.

Brandon et al. (1998), dans une étude réalisée sur les parcs nationaux de l'Amérique latine, ont tiré quelques conclusions intéressantes qui illustrent bien le cas brésilien :

- la majeure partie des défis liés à la conservation de la biodiversité sont politiques ;

- l'efficacité de la gestion des aires protégées dépend de la compréhension du contexte social ;

- la mise en œuvre de solutions pour la conservation de la biodiversité requiert une nouvelle approche conceptuelle, à l'intérieur et à l'extérieur de ces aires.

\section{Défis et enjeux}

Pour répondre à certaines de ces questions et aux défis de la Convention sur la diversité biologique, le Plan stratégique national pour les aires protégées (PNAP) a été établi en 2006, à la suite d'une série de discussions au niveau national entre des acteurs gouvernementaux, des représentants de la société civile et des interlocuteurs du milieu académique et privé. L'objectif central du PNAP est d'orienter le processus d'implantation et de gestion des aires protégées sur le territoire national, en considérant les défis liés à la mise en place d'une gouvernance démocratique, pour des scénarios à moyen et long terme.

Cette évolution illustre avec clarté le changement d'approche des politiques publiques, à partir du constat qu'il n'est plus possible de continuer à penser des stratégies de conservation de la nature dissociées de la dynamique socio-économique régionale et en considérant la réalité d'un pays caractérisé par de fortes inégalités sociales. De la même manière, le PNAP a montré l'urgence de l'intégration de politiques publiques de 
conservation et de développement, dans une logique de partenariat entre les trois sphères gouvernementales, et également entre le gouvernement et la société. Il est clair que, dans cette logique, la gestion des parcs nationaux implique un regard au-delà de ses limites constitutives, avec une perspective d'intégration dans l'ensemble des politiques publiques. Ce mouvement requiert évidemment une pratique de dialogue transversal et intersectoriel, à commencer par l'exercice de l'intégration des conseils de gestion des parcs nationaux dans les conseils de développement municipaux et les comités locaux de l'Agenda $21^{8}$. Pour cette nouvelle pratique, il est essentiel que la qualité de fonctionnement de ces conseils ainsi que leur efficacité en matière de gouvernance démocratique soient évaluées. Il est également fondamental de considérer la nécessité de dialogue entre les différents acteurs gouvernementaux engagés dans les conflits les plus récurrents pour la gestion de parcs nationaux, en particulier l'INCRA ${ }^{9}$ et la FUNAI ${ }^{10}$.

Dans le contexte actuel de la gestion des parcs nationaux, leur superposition avec les terres indigènes et la pression de l'occupation de la zone périphérique par l'agriculture ou l'élevage développés par de grands et petits propriétaires ruraux constituent des questions clés. Il faut également considérer que, dans le cas brésilien, les terres indigènes ne font pas partie du Système national des unités de conservation (SNUC). De plus, elles ne sont pas soumises au même cadre institutionnel et légal que les aires protégées, sous la responsabilité de l'Institut Chico Mendes, lié au ministère de l'Environnement. D'autre part, les parcs nationaux subissent une forte pression des actions de la réforme agraire, officiellement développées par l'INCRA et en parallèle promues par le Mouvement des Sans Terre (MST), qui ont tendance à considérer les parcs nationaux comme des vides démographiques à occuper. Les parcs nationaux qui font partie de biomes, comme le Cerrado et l'Amazonie, subissent également la forte pression du développement de monocultures, comme le soja, en fonction de sa valeur sur le marché international et de sa contribution au produit intérieur brut (PIB). Par contre, dans le cas de la forêt atlantique, la pression sur les ressources naturelles a pour origine principale la déforestation du fait de l'expansion urbaine, la spéculation immobilière et le tourisme, en particulier sur l'axe Rio de Janeiro - São Paulo. A ces questions on peut également ajouter le problème foncier au niveau national, du fait que la majorité des parcs nationaux n'ont pas une situation foncière régularisée, ce qui ne sera probablement pas résolu à court ou moyen terme.

Pour une projection de scénarios futurs, il est important de prendre également en considération les priorités de politiques publiques liées au Plan d'accélération de croissance (PAC) qui inspire un débat national sur l'opposition entre développement et protection de la nature. Le PAC établit des priorités pour les investissements stratégiques en termes d'infrastructure et d'énergie (biodiesel/biocarburants au premier chef). Le tourisme a également récemment émergé dans la politique publique comme vecteur d'inclusion sociale.

De la même manière, il est essentiel que la perspective pour la gestion des parcs nationaux transcende l'approche locale, pour atteindre une vision écosystémique régionale et l'application des politiques d'aménagement du territoire ${ }^{11}$. Jusqu'à maintenant, la gestion s'est focalisée sur les problèmes internes des parcs et se limitait au contrôle de l'utilisation des ressources naturelles renouvelables selon une approche quasi-policière, ou se limitait à la discussion des questions urgentes au niveau des conseils de gestion. Mais l'intégrité de ces espaces protégés ne sera pas assurée si la gestion n'est pas capable de considérer la dynamique de développement régional au 
niveau des politiques publiques, fréquemment contradictoires, sur le même territoire. À ce sujet, la stratégie brésilienne de protection de la nature a récemment privilégié une approche systémique, à partir de la conception stratégique de mosaïques ou de corridors de biodiversité, dans lesquels les espaces protégés sont perçus selon une perspective régionale.

Un autre défi qui s'impose à la gestion des parcs nationaux provient des différences culturelles liées à la perception de la nature. Le PNAP intègre la protection de la biodiversité et également de la socio-diversité. Ceci amène directement à la question des subjectivités et symbolismes impliqués dans le processus de protection de la nature. Cependant, les études sur l'interprétation symbolique du patrimoine naturel et culturel sont encore rares dans la recherche en sciences humaines et sociales et dans la pratique de gestion des parcs nationaux. La gestion des parcs nationaux requiert non seulement la connaissance des espèces de la flore et de la faune mais également de la diversité culturelle et des différentes formes d'interprétation et d'appropriation du patrimoine naturel par les populations locales. Cette conception implique une nouvelle forme d'action, capable d'accepter et de mettre en valeur le "protagonisme social» pour la gestion de la biodiversité, ce qui n'est pas toujours évident dans les modèles classiques de protection de la nature. Ce mouvement implique la consolidation des notions de patrimoine collectif (dans une perspective intergénérationnelle), la construction de pactes sociaux et des canaux efficaces pour la négociation. Plus spécifiquement, la gestion des parcs nationaux demande un profil différencié de gestionnaire, plus flexible devant les demandes des différents acteurs et secteurs sociaux et basée sur une formation permanente en gestion de conflits et communication sociale.

Par ailleurs, un des défis centraux du PNAP pour la gestion de parcs nationaux est la répartition équitable des coûts et des bénéfices associés à la protection de la biodiversité. Jusqu'à présent les pratiques de gestion se sont concentrées sur des stratégies de " commande et contrôle ", ce qui tend à écarter les acteurs sociaux de la responsabilité de tout ce qui est considéré comme bien public. Tant que les parcs nationaux ne seront pas interprétés par les populations locales comme un patrimoine collectif mais uniquement comme un bien public distant de leur réalité quotidienne et toujours associé à la restriction d'usage des ressources, des avancées en matière de gestion participative de la biodiversité restent peu probables. De plus, dans la complexité d'un pays en voie de développement, le succès des stratégies de gestion, pour les groupes humains qui habitent à l'intérieur ou à la périphérie du parc national, est directement lié à la génération de bénéfices économiques et sociaux.

Ainsi, un des défis majeurs pour la gestion des parcs nationaux sera de penser ces espaces comme une opportunité pour la construction de modèles de développement régional, basés sur la valorisation de la nature comme stratégie d'intégration sociale. Dans ce cadre, l'écotourisme, et toute sa chaîne de biens et de services, émerge comme une des principales alternatives, à moyen et long terme, pour la majorité des parcs nationaux brésiliens. Ceci pourra se faire si les politiques de tourisme, de protection de la nature et d'intégration sociale sont associées dans l'action gouvernementale.

41 Cependant, la question centrale pour l'avenir est liée au "protagonisme social » dans la politique publique. Pour l'instant, il n'est pas encore possible d'avoir une évaluation de l'efficacité des instances participatives comme outils de gouvernance démocratique. Mais, à moyen terme, il est fondamental que s'évalue le fonctionnement de ces instances depuis leur origine. Il faut également consolider les méthodologies et directives participatives 
pour leur mise en place et leur évaluation. De la même manière, dans un contexte hétérogène comme c'est le cas au Brésil, un grand effort doit être fait sur la formation permanente des conseillers, pour que les conseils puissent accomplir leurs fonctions, non seulement comme des instances formelles du SNUC, mais comme des mécanismes effectifs de participation sociale dans les politiques publiques. Il faut enfin se rappeler qu'une réelle participation sociale dans les instances prévues par le SNUC dépendra directement d'une évolution des habitudes de gestion, historiquement centralisées et formatées selon des modèles cartésiens d'administration publique.

\section{Conclusion}

Le Plan stratégique national des aires protégées (PNAP) converge clairement vers les objectifs de la Convention sur la diversité biologique et les principes de l'Accord de Durban (IUCN 2003). Cet accord oriente les gouvernements et les sociétés vers des actions positives en faveur des aires protégées. Il propose également l'élaboration d'un nouveau paradigme pour la gestion de la biodiversité, impliquant des actions de promotion et de synergie entre la conservation de la biodiversité, la gestion des systèmes supports de la vie et la promotion du développement durable.

$\mathrm{Au}$ Brésil, ce défi s'exprime à travers la construction d'un modèle de protection de la nature avec une identité nationale, qui va exiger de toute la société un grand effort de remise en cause des concepts et procédures, ainsi que la déconstruction des présupposés résultant de la perspective historique du «mythe moderne de la nature inviolée », dont les parcs nationaux constituent le principal symbole.

Merci à Jean-Pierre Briot pour ses commentaires détaillés sur ce texte.

\section{BIBLIOGRAPHIE}

Bensusan N. 2002 - Seria melhor mandar ladrilhar? Biodiversidade : como, para que e porquê ? Brasília, UNB - Instituto Socioambiental, $252 \mathrm{p}$.

Bensusan N. 2006 - Conservação da Biodiversidade em áreas protegidas. Rio de Janeiro, Fundação Getúlio Vargas, $176 \mathrm{p}$.

Brandon K., Redfort K.H. \& Sanderson S.E. (Ed.) 1998 - Parks in peril: people, politics, and protected areas. Washington D.C., Island Press, 519 p.

BRASIL 2000 - Lei N 9.985 de 18 de julho de 2000 que institui o Sistema Nacional de Unidades de Conservação. Brasília, Diário Oficial de 19 de Julho 2000.

BRASIL 2002 - Decreto $N^{\circ} 4.340$ de 23 de agosto de 2002, regulamenta os artigos da Lei $N^{\circ} 9.985$ que institui o Sistema Nacional de Unidades de Conservação, SNUC. Brasília, Diário Oficial de 23 de Agosto 2002.

BRASIL 2006 - Decreto N 5.758 de 13 de abril de 2006 que institui o Plano Estratégico Nacional de Áreas Protegidas. Brasília, Diário Oficial de 17 de Abril 2006. 
Dias B.F.S. 1994 - O papel das unidades de conservação face à Convenção sobre a Diversidade Biológica e à Constituição Federal de 1988 : uma análise conceitual hierarquizada. Travail non publié.

Diegues A.C. 1996 - O Mito Moderno da Natureza Intocada. São Paulo, Ed. Hucitec, 169 p.

Dourojeanni M.J. \& Pádua M.T. 2001 - Biodiversidade : A hora decisiva. Curitiba, Editora da UFPR, $284 \mathrm{p}$.

IBAMA 2006 - Instituto Brasileiro do Meio Ambiente e dos Recursos Naturais Renováveis, « http://www.ibama.gov.br» (accédé le 15/01/2006).

Irving M.A. 2000 - Diplomacia contemporanea e meio ambiente : o contexto brasileiro. In Mendes Silva R. \& Brigagão C. (Ed.), História das Relações Internacionais do Brasil. Rio de Janeiro, CEBRI, $582 \mathrm{p}$.

Irving M.A. 2002 - Refletindo sobre o ecoturismo em áreas protegidas : tendencias no contexto brasileiro. In Irving M.A. \& Azevedo J. (Ed.), Turismo: o desafio da sustentabilidade. São Paulo, Futura : 47-68.

Irving M.A, Cozzolino L.F., Fragelli C. \& Sancho A. 2006 - Construção de governança democrática : interpretando a gestão de parques nacionais no Brasil. In Irving M.A. (Ed.), Áreas Protegidase Inclusão Social : construindo novos significados. Rio de Janeiro, Aquarius : 41-75.

IUCN 2003 - Acuerdo de Durban, V Congresso Mundial de Parques da IUCN, 8-17 septembre 2003, Durban, Afrique du Sud.

Medeiros R., Irving M.A. \& Garay I. 2004 - A proteção da natureza no Brasil : Evolução e conflitos de um modelo em construção. Revista de Desenvolvimento Econômico 7(1): 83-93.

Pádua J.A. 2004 - A ocupação do território brasileiro e a conservação dos recursos naturais. In Takahashi L.Y., Milano M.S. \& Nunes M.L. (Org.), Unidades de Conservação : atualidades e tendências. Curitiba, Editora da Fundação 0 Boticário de Proteção à Natureza, 208 p. (v. 1).

UNEP/CBD 2005 - Towards effective protected areas systems: An action guide to implement the Convention on Biological Diversity. Montreal, Canada, Secretariat of the Convention on Biological Diversity, CBD Series 18, $105 \mathrm{p}$.

Van Schaik C. \& Rijksen H.D. 2002 - Projetos integrados de conservação e desenvolvimento : Problemas e Potenciais. In Terborgh J., Van Schaik C., Davenport L. \& Rao M. (Ed.), Tornando os parques eficientes : estratégias para a conservação da natureza nos trópicos. Curitiba, Editora da UFPR/ FBPN : 37-52.

\section{NOTES}

1. Les Unités de conservation (Unidades de Conservação: UC, dans la terminologie brésilienne) considèrent les aires formellement délimitées pour la protection, à l'exception des terres indigènes (indiens) et quilomboles (descendants d'esclaves).

2. Loi N 9.985 (Brasil 2000) et Décret 4.340 (Brasil 2002).

3. Décret 5.758 (Brasil 2006).

4. Créé en 1872.

5. Bien que la première proposition de création de parcs nationaux date de 1876 (Bensusan 2006).

6. Avec la participation équilibrée du pouvoir public et des différents secteurs de la société.

7. Dans le cas des parcs nationaux, les conseils ont un caractère uniquement consultatif.

8. Également considérés comme prioritaires par le gouvernement. 
9. Institut National de Colonisation et de Réforme Agraire, parce qu'un des problèmes les plus sérieux pour la gestion des espaces protégés est lié à la question de la terre en milieu rural.

10. Fondation nationale de l'Indien, important acteur gouvernemental à cause de certains recouvrements entre Unités de conservation et Terres indigènes.

11. Dans cette logique, le Plan national d'aménagement du territoire (PNOT) et la Politique nationale des ressources hydriques constituent les points focaux pour l'articulation avec les politiques de protection de la nature.

\section{RÉSUMÉS}

Les parcs nationaux constituent des aires protégées stratégiques, au niveau global, pour la conservation de la biodiversité, du fait de leur reconnaissance et de leur diffusion internationale. Ils illustrent, comme aucun autre type d'espace protégé, les défis pour la gestion de la biodiversité. Ces défis résultent de la séparation historique nature/société, caractéristique des sociétés modernes, en particulier dans le cas des pays en voie de développement dotés d'une importante diversité biologique. Cet article a pour objectif de présenter et d'analyser le contexte et les défis en matière de politiques publiques pour la gestion des parcs nationaux au Brésil, en considérant les engagements du pays pour la Convention sur la diversité biologique. Il illustre notamment la tension entre conservation de la nature et dynamique socio-économique, à partir d'une approche historique et du cadre actuel d'application des politiques publiques.

National Parks represent strategic protected areas for biodiversity conservation, at the global level, given their international recognition and promotion. More than any other type of protected area, they illustrate the challenges of biodiversity management. These challenges are the result of the historical nature/society segregation, characteristic of modern societies, especially in the case of developing countries with great biological diversity. The present paper aims at presenting and discussing the context and challenges of public policies in the case of Brazilian National Parks, considering the country's commitments to the Biological Diversity Convention. It illustrates the tension between nature conservation and socio-economic dynamics, from a historical perspective and on the basis of the current framework of public policies' implementation.

\section{INDEX}

Mots-clés : parcs nationaux, politiques publiques, biodiversité, inclusion sociale

Keywords : National Parks, public policies, biodiversity, social inclusion, Brazil

Index géographique : Brésil 


\section{AUTEUR}

MARTA DE AZEVEDO IRVING

École Doctorale Eicos/IP, Université Fédérale de Rio de Janeiro (UFRJ)

Rua Fernando Ferrari, 61/502, Botafogo, Rio de Janeiro, RJ 22231-040 Brésil

mirving@mandic.com.br 